\title{
On the Wideband EMI Response of a Rotationally Symmetric Permeable and Conducting Target
}

\author{
Lawrence Carin, Fellow, IEEE, Haitao Yu, Yacine Dalichaouch, Alexander R. Perry, Senior Member, IEEE, \\ Peter V. Czipott, and Carl E. Baum, Fellow, IEEE
}

\begin{abstract}
A simple and accurate model is presented for computation of the electromagnetic induction (EMI) resonant frequencies of canonical conducting and ferrous targets, in particular, finite-length cylinders and rings. The imaginary resonant frequencies correspond to the well known exponential decay constants of interest for time-domain EMI interaction with conducting and ferrous targets. The results of the simple model are compared to data computed numerically, via method-of-moments (MoM) and finiteelement models. Moreover, the simple model is used to fit measured wideband EMI data from ferrous cylindrical targets (in terms of a small number of parameters). It is also demonstrated that the general model for the magnetic-dipole magnetization, in terms of a frequency-dependent diagonal dyadic, is applicable to general rotationally symmetric targets (not just cylinders and rings).
\end{abstract}

Index Terms-Induction, magnetization, resonance, sensing.

\section{INTRODUCTION}

$\mathbf{E}$ LECTROMAGNETIC induction (EMI) is widely used for the detection and discrimination of conducting and ferrous targets. In the context of subsurface sensing, EMI is used to sense buried metal mines as well as unexploded ordnance (UXO) [1], [2]. Since the conductivity of such targets is typically many orders of magnitude larger than the conductivity of soil, the target can usually be analyzed in free space. We consider such an analysis here. Most land mines and UXO exhibit rotational symmetry, with this symmetry yielding a simplification of the EMI phenomenology. In particular, as discussed further in Section II, the magnetization tensor of such targets becomes diagonal (with regard to a coordinate system with an axis along the target axis of symmetry). Moreover, we demonstrate that each element of the diagonal corresponds to a magnetic dipole moment, oriented either parallel to or orthogonal to the target axis.

The wideband frequency-domain EMI response of a conducting and ferrous target is characterized by EMI resonant frequencies, which exist along the imaginary axis of the

Manuscript received June 18, 2000; revised October 27, 2000. This work was supported in part by Strategic Environmental Research and Development Program Contract DACA72-99-C-0012-CU-1123, a U.S. Army Research Office Demining MURI, and by the Air Force Office of Scientific Research.

L. Carin and H. Yu are with the Department of Electrical and Computer Engineering, Duke University, Durham, NC 27708-0291 USA.

Y. Dalichaouch, A. R. Perry, and P. V. Czipott are with Quantum Magnetics, San Diego, CA 92192 USA.

C. E. Baum is with the Air Force Research Laboratory, Directed Energy Directorate, Kirtland Air Force Base, Albuquerque, NM 87108 USA.

Publisher Item Identifier S 0196-2892(01)04840-9. complex-frequency plane [3]. Note that here we use the $\omega$ plane for complex frequencies, instead of the $s=\Omega+j \omega$ plane as in the two-sided Laplace transform [4]. Assuming an $\exp (j \omega t)$ time dependence, a purely imaginary resonant frequency corresponds to the exponential decay characteristic of time-domain EMI interaction with conducting and ferrous targets. As is discussed in Section II, the imaginary resonant frequencies corresponding to magnetic-dipole modes play an important role in characterizing the frequency and time-domain properties of the magnetization tensor.

The magnetization tensor and EMI resonances have been discussed previously [4]. The principal contribution of this paper is a recognition that the EMI resonant modes of high-conductivity targets, characterized by purely imaginary resonant frequencies, are analogous to the resonant modes of high-permittivity, low-loss microwave resonators [5], which resonate at nearly purely real resonant frequencies. Once this duality is understood, all of the many techniques developed in the microwave community for characterization of high-permittivity dielectric resonators [6]-[8] (operating at nearly real frequencies) can be directly transitioned to the EMI high-conductivity, high-permeability problem (with resonant frequencies at imaginary frequencies). Moreover, this understanding yields an explicit interpretation of the EMI resonant modes in terms of dipole and higher order magnetic moments, from which the physical significance of particular EMI modes can be accrued.

Many metal land mines and UXO are composed of parts that resemble finite-length cylinders and rings and therefore, the EMI resonant modes of such targets are of interest. Such targets, as well as more-general shapes, can be analyzed via numerical algorithms, such as the method of moments (MoM) [3]. Unfortunately, such calculations are very expensive computationally, since the size of the MoM basis functions typically must be small relative to the skin depth [3]. This requirement significantly limits the size of the targets that can be considered, and results in significant computer run times, and computer memory requirements. However, for simple but important shapes, such as finite-length cylinders and rings, one can make use of the duality between EMI resonances and microwave resonances to yield very simple but highly accurate approximate models. In particular, as detailed in Section II, we have employed these simple models to calculate the (imaginary) resonant frequencies of conducting cylinders and rings, with the computed data compared favorably to data simulated numerically. In those examples we restrict ourselves to relatively small targets, for which the numerical analysis is tractable. The approximate model is applicable to cylinders and 
rings of arbitrary size. A long conducting and ferrous cylinder, for example, provides a good representation of a UXO, and therefore we can use our simple model to predict the imaginary resonant frequencies (time-domain decay constants) of such targets, as a function of material parameters (conductivity and permeability).

The remainder of the paper is organized as follows. In Section II, we explain the duality between conducting and ferrous targets at EMI frequencies and high-permittivity resonators at microwave frequencies. We also elucidate how to transition approximate models from the microwaves community, for calculation of the EMI resonant frequencies of finite-length cylinders and rings. In Section III, we compare the resonant frequencies computed via the approximate model with those computed by rigorous numerical algorithms. A demonstration of how the model is used to characterize measured data is discussed in Section IV, in which we investigate the measured wideband EMI response from ferrous cylinders. Conclusions from this work are discussed in Section V.

\section{FundAMENTAL THEORY}

\section{A. Resonant EMI Model}

Consider an arbitrarily shaped highly (but not perfectly) conducting and possibly permeable target, residing in free space. At induction frequencies [1], [2], the displacement current can be neglected and the wavenumber $k$ inside the target satisfies $k^{2}=-j \omega \mu_{o} \mu_{r} \sigma$, where $\mu_{o}$ is the free-space permeability, $\mu_{r}$ is the relative permeability, $\sigma$ is the conductivity, and $\omega$ is the angular frequency. The source-free fields inside the target satisfy

$$
\nabla^{2} \boldsymbol{\Phi}-j \omega \mu_{o} \mu_{r} \sigma \boldsymbol{\Phi}=0
$$

where $\boldsymbol{\Phi}$ denotes either the electric or magnetic fields. This source-free wave equation can be solved for the resonant modes of the target, from which the target resonant frequencies and fields can be computed. A similar analysis is commonly performed for microwave low-loss dielectric resonators, for which $k^{2}$ is nearly purely real (resonators with a high quality factor [5]), as is the resonant frequency. For the EMI frequencies of interest here, we note that $k^{2}$ is positive real when the frequency $\omega$ is positive imaginary. For the $\exp (j \omega t)$ time dependence assumed, this implies that the EMI resonant modes decay exponentially with time. This is consistent with the well known late-time characteristics of transient EMI excitation of conducting and permeable targets [1].

The computation of the real resonant frequencies of microwave dielectric resonators is of significant interest for filter design, and therefore there is a vast literature on this subject [5]-[8]. Moreover, van Bladel [5] has developed an elegant theory for the modes of microwave resonators of very high permittivity, with this directly transferable to the EMI resonances of highly (but not perfectly) conducting and permeable targets. Of more importance from a practical standpoint, simple algorithms have been developed for calculation of the resonant modes of high-permittivity rotationally symmetric dielectric resonators [6]-[8], with that previous work transitioned here for characterization of the EMI modes of rotationally symmetric
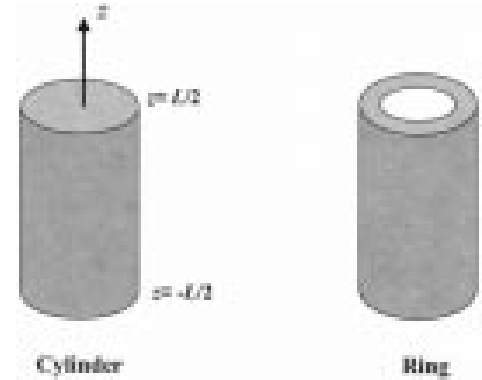

Fig. 1. Cylinder and ring targets for which the approximate model in Section II-B is developed, each highly conducting and possibly ferrous.

highly conducting and permeable targets. While the restriction to rotationally symmetric targets is clearly a limitation, such are of interest for many conducting and permeable land mines and unexploded ordnance (UXO) [1], [2].

\section{B. Finite-Length Cylinder or Ring Target}

We develop below a simple model for the EMI response of general conducting and permeable rotationally symmetric targets. However, we initially limit the discussion to the case of a finite-length cylinder or ring (Fig. 1), for which the model parameters can be computed explicitly. To analyze the resonant characteristics (with purely imaginary resonant frequencies) of finite-length cylinders or rings, we first consider such targets of infinite length, followed by imposition of appropriate boundary conditions at $z= \pm L / 2$ (see Fig. 1). As discussed above, for purely imaginary frequencies, the infinite-length conducting and permeable cylinder or ring behaves analogous to an infinite-length low-loss dielectric waveguide (when the low-loss waveguide operates at real frequencies). If the target axis is oriented along the $z$ axis of a cylindrical coordinate system, the rotational symmetry of the targets dictates a $\cos (m \phi)$ azimuthal variation for the waveguide modes, where $m$ is an integer greater than or equal to zero. The lowest-order modes have no azimuthal variation, corresponding to $m=0$. The $m=0$ modes reduce to two classes [9]. Those with electric fields transverse to the $z$ direction (TE) and those with magnetic fields transverse to $z$ (TM). The resonant modes corresponding to the TM modes can be found by replacing the boundary of the target by a magnetic wall [5], [6] and letting $\beta^{\mathrm{TM}}$ represent the longitudinal wavenumber for a TM mode (infinite waveguide). The $n$th corresponding resonant mode, for a cylinder or ring target of length $L$, resonates at the imaginary frequency for which the wavenumber $\beta^{\text {TM }}$ satisfies

$$
\beta^{\mathrm{TM}} L=2 \pi n \quad n=1,2,3, \ldots
$$

For the TE modes, the simple magnetic-wall approximation at the ends of the target is not sufficiently accurate, and therefore we utilize a higher-order approximation [6]. In particular, we note in Fig. 2 that the cylindrical or ring target has a symmetry plane at $z=0$, with this represented by a short-circuit or opencircuit plane, depending on the mode of interest. The mode that resonates at the lowest frequency corresponds to a short circuit [6], so we consider that case. Let $\beta^{\mathrm{TE}}$ denote the longitudinal wavenumber for the TE modes supported by the infinite-length cylinder or ring. The impedance seen from $z=L / 2$, looking 


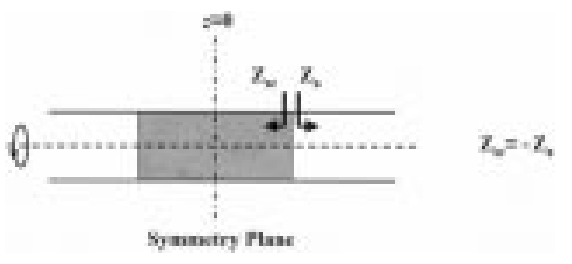

Fig. 2. Schematic of the resonance condition applied for computation of the resonant frequencies of the TE modes supported by the finite-length cylinders and rings considered.

toward the short-circuit symmetry plane is defined as $Z_{s c}$, while the impedance seen at $z=L / 2$ looking toward the air region is denoted $Z_{a}$, where (using magnetic walls)

$$
\begin{gathered}
Z_{s c}\left(\omega=j \omega_{i}\right)=-\frac{\omega_{i} \mu_{o} \mu_{r}}{\beta^{\mathrm{TE}}} \tan \left(\beta^{\mathrm{TE}} L / 2\right) \\
Z_{a}\left(\omega=j \omega_{i}\right)=-\omega_{i} \mu_{o} / \sqrt{k_{c}^{2}+\omega_{i}^{2} \mu_{o} \varepsilon_{o}}
\end{gathered}
$$

and $k_{c}$ is the cutoff wavenumber for the TE mode under consideration. The $n$th purely imaginary resonant frequency occurs under the condition $Z_{s c}=-Z_{a}[6]$, and there are an infinite number of solutions to this transcendental equation (corresponding to the infinite number of such modes with short-circuit symmetry).

A multipole expansion of the fields associated with the above TE and TM modes yields important insight into the fields radiated by these source-free modes. It is straightforward to demonstrate that the lowest-order moment associated with the $m=0$ (no azimuthal variation) TE resonant modes corresponds to a magnetic dipole oriented along the $z$ direction. By contrast, the lowest-order moment associated with the $m=0 \mathrm{TM}$ resonant modes corresponds to a magnetic quadrupole (equivalently, an electric dipole [5]). Consequently, to a sensor that measures the magnetic field, the $m=0 \mathrm{TM}$ resonant modes contribute negligibly vis-a-vis the TE modes.

To account for the resonant modes with a dipole magnetic moment oriented orthogonal to the $z$ axis, we must consider the $m=1$ waveguide modes, with the associated physical significance discussed subsequently. Unlike the $m=0$ waveguide modes, for infinite cylindrical or ring waveguide, the $m=1$ modes are not simply TE or TM, but are hybrid modes corresponding to a superposition of these two simpler mode types [9]. Consequently, to determine the resonant frequencies of the $m=1$ modes, we first numerically compute the longitudinal wavenumber for the corresponding infinite-length waveguide, denoted $\beta^{E M}$ for the hybrid modes [9]. The imaginary resonant frequencies are then computed using a relationship analogous to (2). For the hybrid modes one cannot easily define a modal impedance, and therefore we cannot employ a more-sophisticated resonant condition, as in (3). Nevertheless, as demonstrated below, the accuracy of the resonant frequencies computed via this simple model are in very good agreement with data computed via a rigorous numerical model. Finally, through consideration of the $m=1$ modal fields, it is easily shown that the lowest-order multipole term corresponds to a magnetic dipole oriented perpendicular to the axis of rotation.

Summarizing, we have demonstrated that the lowest-order resonant modes of a finite-length highly conducting and perme-
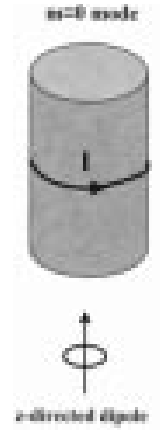
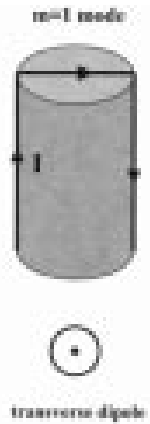
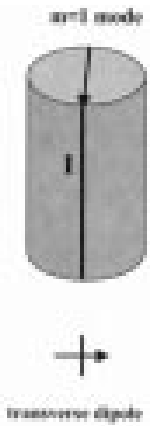

Fig. 3. Schematic of the currents associated with the magnetic dipoles EMI-excited on a conducting and possibly ferrous target. The dipole oriented along the $z$ axis corresponds to the $m=0$ TE modes, and the transverse magnetic dipole moments correspond to the $m=1$ hybrid modes.

able cylindrical or ring target correspond to magnetic dipoles oriented along and perpendicular to the target axis. Each of these resonant modes resonates with a purely imaginary resonant frequency. The modes with magnetic dipole oriented along the target axis correspond to the $m=0$ TE modes, while the magnetic moments oriented orthogonal to the axis correspond to the $m=1$ hybrid modes. The explicit imaginary resonant frequencies can be computed easily using the simple expressions reviewed above, these borrowed from the microwave community [5]-[8]. In Fig. 3, we schematically plot the currents characteristic of the two mode classes. Note that the dipole moment perpendicular to the target axis corresponds to a current density for which $\boldsymbol{J}(\phi)=-\boldsymbol{J}(\phi+\pi)$, explaining the $\cos (\phi)$ variation.

\section{Target Transfer Function}

The resonant frequencies corresponding to each EMI resonant mode are manifested as poles in the complex frequency plane [3], [4], where as discussed above all EMI poles reside on the imaginary frequency axis. Recalling that the $m=0 \mathrm{TE}$ modes correspond to dipoles oriented in the $z$ direction, and the $m=1$ hybrid modes correspond to dipoles perpendicular to $z$, the frequency-dependent dyadic dipole magnetization is expressed as

$$
\begin{aligned}
\boldsymbol{M}(\omega)= & \boldsymbol{z} \boldsymbol{z}\left[m_{z}(0)+\sum_{k} \frac{\omega m_{z k}}{\omega-j \omega_{z k}}\right] \\
& +(\boldsymbol{x} \boldsymbol{x}+\boldsymbol{y} \boldsymbol{y})\left[m_{p}(0)+\sum_{i} \frac{\omega m_{p i}}{\omega-j \omega_{p i}}\right] .
\end{aligned}
$$

The frequency-independent parameters $m_{z}(0)$ and $m_{p}(0)$ account for the dipole moments contributed by ferrous targets, with these constants equal to zero for nonpermeable targets. In (4) we sum over the infinite number of resonant $m=0 \mathrm{TE}$ and $m=1$ hybrid modes, although in practice, a small number of modes are sufficient for representation of the frequency-dependent dyadic dipole magnetization. The quantities $\omega_{z k}$ and $\omega_{p i}$ are real, and therefore, each of the summed terms has a pole on the imaginary frequency axis. Concerning the frequency dependence of each of the summed terms, we note that at high EMI frequencies the resonant mode asymptotically approaches the inductive high-frequency limit [10], for which the real part of the response approaches a constant, while the imaginary part goes 
to zero. Moreover, as the frequency goes to zero, the response of each EMI resonant mode will also go to zero [10] [leaving only the constants $m_{z}(0)$ and $m_{p}(0)$ ]. These two conditions motivate the $\omega$ in the numerator of each summed term. We also note that the relative strengths $m_{z k}$ of the $z$-directed dipole modes are fixed by the target geometry and material properties, with this also true for the relative weights of $m_{p_{i}}$ [11] Finally, the impulse response of the dipole magnetization is easily found via a Fourier analysis of (4), and is given as

$$
\begin{aligned}
\hat{\boldsymbol{M}}(t) & \\
= & z \boldsymbol{z}\left[m_{z}(0) \delta(t)+\frac{\partial}{\partial t} \sum_{k} u(t) m_{z k} \exp \left(-\omega_{z k} t\right)\right] \\
& +(\boldsymbol{x} \boldsymbol{x}+\boldsymbol{y} \boldsymbol{y})\left[m_{p}(0) \delta(t)+\frac{\partial}{\partial t} \sum_{i} u(t) m_{p i} \exp \left(-\omega_{p i} t\right)\right]
\end{aligned}
$$

Such that real time-domain waveforms are realized, (5) implies that $m_{z}(0), m_{p}(0), m_{z k}$, and $m_{p i}$ must be real quantities.

If we consider an incident, frequency-dependent magnetic field $\boldsymbol{H}^{i n c}(\omega)$, then the magnetic fields induced by a target described by the $\boldsymbol{M}(\omega)$ in (4) are given as [9]

$$
\boldsymbol{H}(\omega)=-\frac{1}{4 \pi} \nabla \times\left[\boldsymbol{M}(\omega) \cdot \boldsymbol{H}^{i n c}(\omega) \times \nabla \frac{1}{r}\right]
$$

where $r$ is the distance from the target dipoles to the observation point. A similar, convolutional relationship holds for time-domain excitation.

\section{General Rotationally Symmetric Targets}

In (4) and (5), we have presented the frequency and time-dependent dipole magnetization for a conducting and permeable finite-length cylinder or ring. In particular, we have demonstrated that these expressions are characterized by the purely imaginary resonant frequencies of the targets, with explicit tools for their approximate calculation presented in Section II-B. We note, however, from the discussion of Section II-A, that any finite size conducting and permeable target will be characterized by resonant modes with purely imaginary resonant frequencies. For simple shapes like the cylinder and ring considered above, the imaginary resonant frequencies can be calculated approximately via simple models, while more-general shapes require numerical algorithms. In [3] we discussed a MoM model for general body-of-revolution (BoR) conducting and permeable targets, and in Section III, we discuss a finite-element method (FEM) applicable to general target shapes. Moreover, due to symmetry [4], the dipole magnetization dyadic for a general BoR will be in the same form as (4) and (5), although for general targets one must estimate the model parameters via a numerical model or from measured data. Many targets of interest (mines and ordnance) are bodies of revolution, and therefore the simple models in (4) and (5) are particularly useful.

While beyond the scope of the present BoR computations, we note that the longitudinal/transverse decomposition in (4) and (5) applies to more general metal targets as well. In particular, the same results apply to such bodies with an $N$-fold rotation axis [12] for $N \geq 3$ (i.e., $C_{N}$ symmetry with $N \geq 3$ ). Axial symmetry planes are not required (see [4] ). Our BoR corresponds to $N=\infty$ with axial symmetry planes $\left(\mathrm{O}_{2}\right.$ symmetry).

\section{COMPARISON OF APPROXIMATE AND NUMERICAL DATA}

\section{A. Numerical Models Considered}

Below we compare the accuracy of the EMI resonances computed using the simple models in Section II-B with data computed via rigorous numerical models. We summarize the numerical models, for completeness. For BoR targets a MoM analysis is particularly useful, in which the currents are expanded in terms of azimuthal harmonics with variation $\cos (m \phi)$. For such problems the currents are expanded only on the surface of the generating arc [3]. This approach has two advantages for our applications: 1) the problem complexity simplifies significantly, since the unknowns, for each mode $m$, are only on a two-dimensional (2-D) arc (rather than in three dimensions), and 2) the azimuthal BoR harmonics with index $m$ are directly applicable to the azimuthal modes discussed in Section II-B, allowing a direct comparison of numerical and approximate EMI resonant frequencies. The details of our BoR MoM model are discussed in [3].

While the BoR MoM model is useful and computationally efficient, it is restricted to a limited class of problems. If the target is not a BoR [15], or if it is composed of inhomogeneous material parameters, a more general model is preferable. In this context we have developed a general hybrid model that combines a volumetric finite-element method (FEM) solution with a boundary element method (BEM) [16], [17]. In the FEM-BEM formulation, the fields inside the target are expanded in terms of general volumetric basis functions, and the surface region is represented in terms of generalized surface basis functions. The unknowns therefore exist inside and on the surface of the target, and there is no need to truncate the computational domain in an absorbing boundary region. The details of the FEM-BEM model have been published elsewhere. In particular, the general FEM-BEM formulation is discussed in [16], [17], with the type of surface basis functions employed discussed in [18]. The FEM-BEM and BoR-MoM models are distinct, and their respective accuracy was assured by demonstrating that these two disparate models yielded the same results, when considering problems for which they are both applicable.

\section{B. Finite-Length Cylinder}

Our initial example is for a stainless-steel cylinder, of length $5.08 \mathrm{~cm}$ and $1.27 \mathrm{~cm}$ diameter. We perform a comparison between resonant frequencies computed via the simple approximate model (Section II-B) and via the rigorous BoR-MoM solution. Note that the target size considered is small, especially relative to large ordnance that may be of interest. This is a limitation of any numerical model, including the BoR-MoM. In particular, the currents on the surface of the BoR are expanded in a basis set [3], and the size of the basis functions must be small relative to the skin depth. Since for large targets, the skin depth may be very small relative to other target dimensions (e.g., length), a large number of basis functions may be required to obtain accurate results. Therefore, as the target size increases, 
one realizes a commensurate increase in the required computer memory and run times. In particular, if $N$ basis functions are required to expand the surface currents, the required memory is order $N^{2}$ and the required computer time is also order $N^{2}$. The FEM-BEM solution has similar restrictions. By contrast, the simple models developed in Section II-A have the significant advantage of being applicable independent of the target size. The computational burden of the numerical algorithms is exacerbated when one considers highly permeable targets, and therefore all comparisons to numerically generated data is for nonferrous targets. We address ferrous targets through consideration of measured data, in Section IV.

The imaginary resonant frequencies correspond to zeros of the MoM or FEM determinant [3] when computed along the imaginary frequency axis. In practice, the determinant does not go to zero at the resonant frequencies, due to numerical truncation errors, but the resonant modes occur at sharp dips in the MoM/FEM determinant when viewed as a function of frequency (see Figs. 4 and 5). In Fig. 4 we plot the determinant of the MoM impedance matrix, assuming the stainless-steel conductivity $\sigma=1.3 \times 10^{6} \mathrm{~S} / \mathrm{m}$, for the $m=0$ and $m=1$ modes. Recall that the $m=0$ modes are in the form of TE and TM modes. The TM modes correspond to magnetic quadrupoles, and therefore are of little interest. However, the MoM solution does not distinguish between TE and TM modes, it calculating the imaginary resonant frequencies of all modes. Consequently, an advantage of the simple EMI solution is its close connection to the underlying physics. Similar information can be obtained from the MoM solution, but one must examine the modal currents to ascertain the mode identity [3]. In addition to plotting in Fig. 4 the determinant of the MoM matrix, we tabulate the imaginary resonant frequencies computed via the approximate model, for the first few magnetic-dipole modes.

In Fig. 4(a) the MoM and approximate model agree to less than $5 \%$ error while in Fig. 4(b) the error is typically less than $2 \%$. Given the simplicity and computational efficiency of the approximate model, such accuracy is good. The approximate results in Fig. 4(b) are a little better than those in Fig. 4(a) because the $m=$ 0 modes are computed using a magnetic wall for the sides of the TE-mode waveguide, while for the hybrid modes characteristic of the $m=1$ modes, we numerically evaluate the modal propagation constant [9] without any such approximation. The $m=0$ approximate solution in Fig. 4(a) are improved slightly if one computes the TE waveguide propagation constant numerically (without a magnetic wall), as was done for the hybrid modes.

Note that, for this example, the $m=1$ mode has a lower fundamental resonant frequency than the $m=0$ mode; we have consistently found this to be true for the nonferrous cylinders examined. The MoM results, for computation of the determinant over the range of imaginary frequencies considered, requires on the order of one hour of CPU time on a $500 \mathrm{MHz}$ Pentium III personal computer. On the same computer, the approximate model requires approximately $20 \mathrm{~s}$.

\section{Finite-Length Ring}

We next consider a ring of $2.54 \mathrm{~cm}$ length, outer radius 1.27 $\mathrm{cm}$ and a wall thickness of $3.175 \mathrm{~mm}$, composed of aluminum $\left(\sigma=3 \times 10^{7} \mathrm{~S} / \mathrm{m}\right)$. The numerical results are computed via the

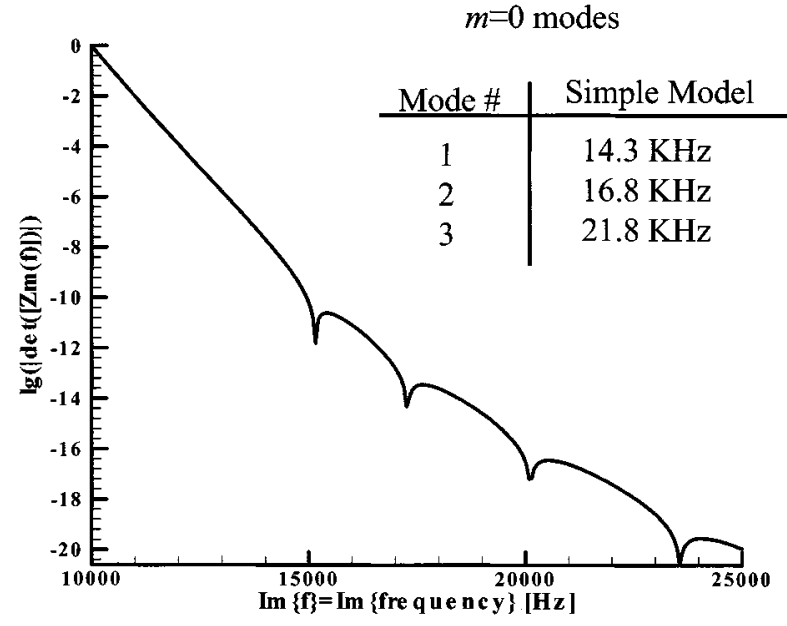

(a)

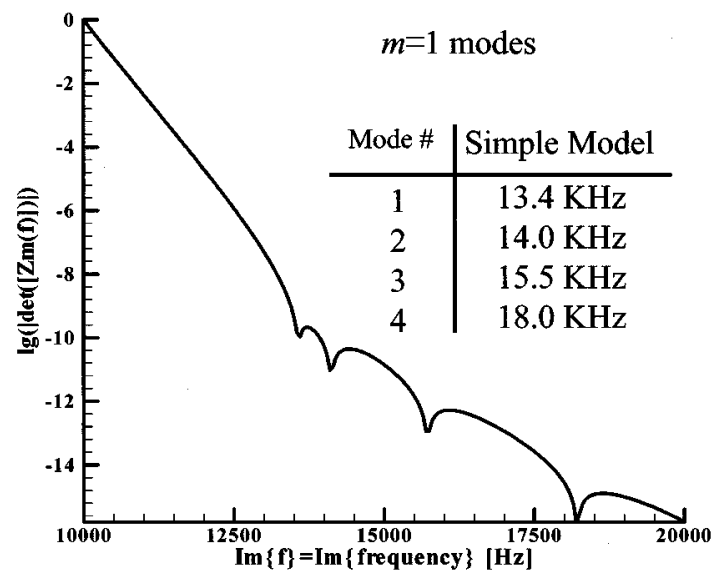

(b)

Fig. 4. Characterization of the magnetic dipoles associated with a cylindrical target of length $5.08 \mathrm{~cm}$ and $1.27 \mathrm{~cm}$ diameter, and conductivity $\sigma=1.3 \times$ $10^{6} \mathrm{~S} / \mathrm{m}$. The curves correspond to the determinant of the MoM impedance matrix. In the inserted table is shown the results from the approximate model in Section II-B. In this context, for the $m=0$ modes we only tabulate results for the magnetic-dipole TE modes; the highest-frequency MoM dip in Fig. 4(a) corresponds to a TM mode (magnetic quadrupole). (a) Modes with magnetic dipole oriented along the target axis and (b) modes with magnetic dipole orthogonal to target axis.

FEM-BEM model, this model applicable to targets of general composition and shape. The disadvantage of this generality is that the FEM-BEM model does not decompose the fields into $\cos (m \phi)$ harmonics, and therefore, a comparison between the approximate and FEM-BEM results is less direct. We therefore compare the accuracy of the approximate solution for the ring mode of lowest resonant frequency, this corresponding to the fundamental $m=1$ mode in the approximate model. As in the BoR-MoM solution, for computation of the numerical resonant frequency, we compute the determinant of an FEM-BEM matrix along the imaginary frequency axis, with the dips corresponding to resonant frequencies. We see in Fig. 5 that the approximate and FEM-BEM solutions agree to within $1.6 \%$. The FEM-BEM solutions, being three-dimensional (3-D), are even more costly computationally than the BoR-MoM solution. While the FEM-BEM solution requires hours of CPU time, on the personal computer discussed above, the approximate solution again requires approximately $20 \mathrm{~s}$. 


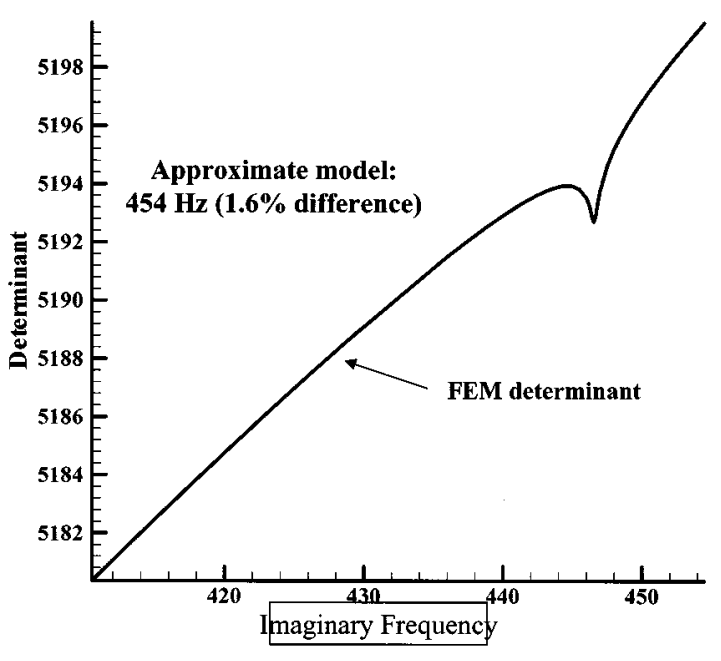

Fig. 5. Characterization of the lowest-order (fundamental) magnetic dipole for a ring target of $2.54 \mathrm{~cm}$ length, outer radius $1.27 \mathrm{~cm}$ and a wall thickness of $3.175 \mathrm{~mm}$, and of conductivity $\sigma=3 \times 10^{7} \mathrm{~S} / \mathrm{m}$. The curve is the determinant of the FEM-BEM impedance matrix, while the approximate model predicted an imaginary resonant frequency of $j 454 \mathrm{~Hz}$.

\section{COMPARISONS TO MEASURED DATA}

\section{A. Sensor Details}

The above comparisons between the approximate model of Section II-B and numerical data computed via the BoR-MoM and FEM-BEM models are encouraging. However, as discussed above, the numerical models have difficulties when considering ferrous targets. Therefore, to consider the accuracy of the model in Section II-B for such targets, we perform a comparison to measured data. The data were taken using a magnetic sensing probe developed by Quantum Magnetics. This probe uses a wide bandwidth room temperature magnetoresistive (MR) sensor operated using a high-performance electronics developed by QM. Unlike induction receiver coils, which measure the rate of change of magnetic field, MR sensors measure the magnetic field itself. As a result, they operate with a frequency-independent response from dc to several $\mathrm{MHz}$. The current sensor operates over the frequency band 0.1 and $400 \mathrm{KHz}$.

\section{B. Comparison of Measured and Theoretical Data}

When considering ferrous targets, there are two parameters that can be varied: the conductivity and permeability. In practice, for a given metal type, there is often a wide range of variability in these parameters, and therefore, it is somewhat misleading to use particular values of $\mu_{r}$ and $\sigma$ in the approximate model (for the resonant frequencies) and compare with measured data. In particular, one can choose $\mu_{r}$ and $\sigma$ such that there is a match, but this does not mean that the $\mu_{r}$ and $\sigma$ used in the model actually correspond to the parameters of that target that was measured. Consequently, we therefore perform an alternative comparison of modeled and measured data. In particular, from (4) and (6) it is clear that the frequency-dependent magnetic field can be expressed in the form

$$
H_{\alpha}(\omega)=a+\sum_{k} \frac{\omega b_{k}}{\omega-j \omega_{k}}
$$

where

$H_{\alpha} \quad$ particular component of magnetic field;

$a$ constant offset due to the ferrous target (zero for a nonferrous target);

$j \omega_{k}$ imaginary resonant modes excited;

$b_{k} \quad$ excitation strengths of the modes, with these dictated by the details of the target and the incident magnetic field.

For the ferrous targets considered below, we consider the optimal match of (7) to the measured data, using just a one-pole model. We therefore perform a least-squared match of (7) to the measured data, where the frequency-independent (constant) free parameters are $a, b_{1}$ and $\omega_{1}$.

We consider a carbon steel pin of $6.858 \mathrm{~mm}$ length and 1.5748 $\mathrm{mm}$ diameter. This target is characteristic of a small firing pin used in plastic antipersonnel land mines. The EMI response was measured over the $0.1-400 \mathrm{KHz}$ band, with the pin axis aligned with the sensor axis (the magnetic dipole moment along the target axis is excited preferentially with this target-sensor orientation, motivating the single-pole model). In Fig. 6, we compare the measured real and imaginary EMI signature (curves) to the EMI model in (7), where the model parameters are shown with points. One notices an excellent match to the computed and measured EMI signature, where here the model parameters employed were $a=-0.1, b_{1}=0.1$ and $f_{1}=8.5 \mathrm{KHz}$ $\left(\omega_{1}=2 \pi f_{1}\right)$.

In practice, while the targets of interest may be rotationally symmetric, the exact conductivity and permeability are generally known only approximately (the same metal type can have substantial variability in these parameters). Therefore, in the context of target classification, it may be best to compute the EMI model parameters from measured data, as above. The extracted model parameters can then be employed in a statistical target-classification algorithm [19], in which the statistical variation of the target-model parameters is accounted for. The comparisons to numerical data in Section III therefore serve to demonstrate the approximate-model accuracy, while in practice the model will typically be applied directly to measured data (as in Fig. 6). The approximate but highly accurate models discussed in Sections II-B and II-C can be used to quickly determine the range of variability in the imaginary resonant frequencies of finite-length cylinder and ring targets, as a function of real-world variability of the target material parameters.

Before closing this section, it is of interest to examine the characteristics of the target in Fig. 6, for the case in which the cylinder axis is orthogonal to the sensor axis. This corresponds to the case for which the orthogonal (to the target axis) magnetic dipoles will be excited preferentially. We employ the same sensor as discussed above, with the comparison between the measured data and the single-pole model shown in Fig. 7. For this case the fitted model parameters in (7) correspond to $a=-$ $0.0114, b_{1}=0.0111$, and $f_{1}=7.6 \mathrm{KHz}$. Note that for this case, the general fit between the measured and model data is good, although not as good as in Fig. 6. The orthogonal magnetic dipole is apparently not excited as strongly, for the EMI response (measured magnetic field orthogonal to the sensor loop) is now an order of magnitude smaller than the results in Fig. 6 


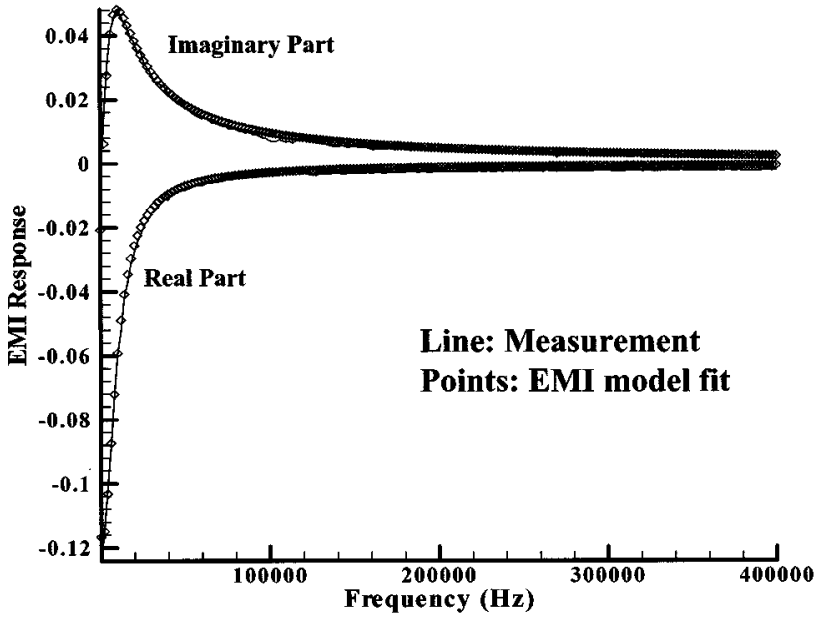

Fig. 6. Magnetic fields measured by an EMI sensor, with the excitation-coil axis aligned with the target axis. The curve corresponds to the measured data, and the points correspond to the fit to the data using a one-pole model, as in (7).

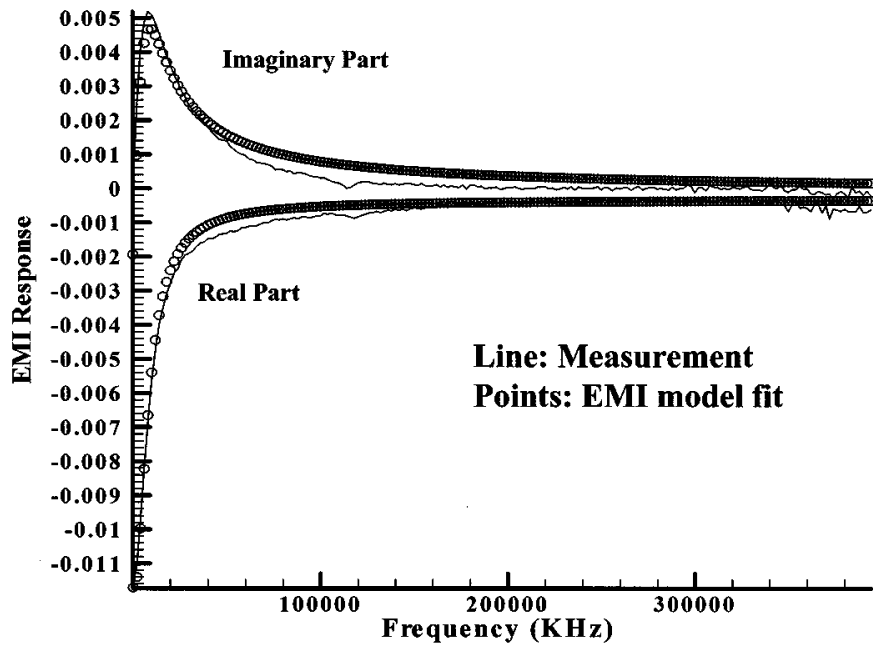

Fig. 7. As in Fig. 6, but now the sensor coil is placed orthogonal to the cylinder axis, aligned with the middle of the cylinder.

(for the same target-sensor distance). The small differences between the measured and model data may therefore be attributed to sensor noise, due to the relatively weak measured signal at this target-sensor orientation. A better fit can also be obtained using a two-pole model.

With regard to the measured data in Figs. 6 and 7, we reiterate that it appears that the magnetic dipole oriented along the target axis is excited more strongly than the dipole orthogonal to this axis. Our theory from Section II can help explain this phenomenon. In particular, the magnetic dipole oriented along the target axis corresponds to an $m=0$ TE mode, while the magnetic dipole orthogonal to this axis corresponds to a higher-order $m=1$ hybrid mode. We therefore attribute the differences in the excitation strengths in Figs. 6 and 7 to relatively stronger excitation of lower order (simpler) modes as compared to weaker excitation of higher order (more complex) modes.

\section{CONCLusions}

The principal contribution of this paper is an explanation of the duality between the response of high-permittivity dielec- tric resonators at microwave frequencies and high-conductivity, high-permeability targets at EMI frequencies. The dielectric resonators resonate at nearly purely real microwave frequencies, while the conducting and ferrous targets have purely imaginary EMI frequencies. An understanding of this duality allows exploitation of the wide range of work that has been done in the microwave community for analyzing high-permittivity resonators. In particular, high-conductivity and high-permeability cylinders and rings are of interest in many targets interrogated via an EMI sensor, and we have transitioned models originally developed in the microwave community to perform an EMI analysis of such targets. The approximate-model EMI results were found to be in close agreement with data computed via rigorous numerical algorithms.

The EMI model, in terms of a diagonal magnetization tensor, in which the EMI resonant frequencies are used explicitly, is applicable to general rotationally symmetric targets, not simply cylinders and rings. This model characterizes the wideband EMI response of such targets in terms of a small number of parameters. This is important in the analysis of measured EMI data which, by exploitation of the simple model, can be fit in terms of a small number of parameters, with these compact parameters employed in a subsequent signal-processing algorithm. We have demonstrated the fit of wideband EMI data, over a $0.1-400 \mathrm{KHz}$ bandwidth, in terms of only three parameters. The target considered was a small ferrous cylinder, characteristic of the firing pin in a plastic land mine. Such fits can be performed with EMI data from more general rotationally symmetric targets.

Future work is required on consideration of more-complex targets, such as actual UXO, that may be composed of multiple parts (e.g., a long cylinder, rings, and possibly a nose composed of a different metal type). Each of these individual components is characterized in terms of its own resonant EMI modes, and it is of interest to examine the degree to which such modes couple in the composite. Moreover, the magnetization tensor is representative of a superposition of various magnetic dipole modes. For a composite target, the different magnetic dipoles from each of the components will in general not be colocated (i.e., the magnetic dipoles associated with a UXO ring may not be located at the same position as the dipoles associated with the larger body). In this context, the dipole magnetization model, in terms of a finite number of magnetic dipoles, must be augmented to consider the location of the dipoles associated with respective subcomponents.

\section{REFERENCES}

[1] Y. Das, J. E. McFee, and R. H. Chesney, "Time domain response of a sphere in the field of a coil: Theory and experiment," IEEE Trans. Geosci. Remote Sensing, vol. GE-22, pp. 360-367, July 1984.

[2] Y. Das, J. E. McFee, J. Toews, and G. C. Stuart, "Analysis of an electromagnetic induction detector for real-time location of buried objects," IEEE Trans. Geosci. Remote Sensing, vol. 28, pp. 278-287, May 1990.

[3] N. Geng, C. E. Baum, and L. Carin, "On the low-frequency natural response of conducting and permeable targets," IEEE Trans. Geosci. Remote Sensing, vol. 37, pp. 347-359, Jan. 1999.

[4] C. E. Baum, Ed., Detection and Identification of Visually Obscured Targets. London, U.K.: Taylor \& Francis, 1998.

[5] J. van Bladel, "On the resonances of a dielectric resonator of very high permittivity," IEEE Trans. Microwave Theory Tech., vol. MTT-23, pp. 199-208, Feb. 1975. 
[6] K. K. Chow, "On the solution and field pattern of cylindrical dielectric resonators," IEEE Trans. Microwave Theory Tech., vol. MTT-14, p. 439, Feb. 1966

[7] M. Verplanken and J. van Bladel, "The electric-dipole resonances of ring resonators of very high permittivity," IEEE Trans. Microwave Theory Tech., vol. MTT-24, pp. 108-112, Feb. 1976.

[8] - "The magnetic-dipole resonances of ring resonators of very high permittivity," IEEE Trans. Microwave Theory Tech., vol. MTT-27, pp. 328-333, Apr. 1979.

[9] R. F. Harrington, Time-Harmonic Electromagnetic Fields: McGraw Hill, 1961.

[10] F. Grant and G. West, Interpretation Theory in Applied Geophysics. New York: McGraw Hill, 1965.

[11] C. E. Baum, "Use of residue and constant-dyadic information in magnetic singularity identification," Air Force Res. Lab., Kirtland AFB, NM, Interaction Note 547, Aug. 1988

[12] C. E. Baum and H. N. Kritikos, "Symmetry in electromagnetics," in Electromagnetic Symmetry, C. E. Baum and H. N. Kritikos, Eds. London, U.K.: Taylor \& Francis, 1995, ch. 1, pp. 1-90.

[13] C. E. Baum, "Symmetry in electromagnetic scattering as a target discriminant," Air Force Res. Lab., Kirtland AFB, NM, Interaction Note 523, Oct. 1996

[14] _ "Application of symmetry to magnetic-singularity identification of buried targets," Air Force Res. Lab., Kirtland AFB, NM, Interaction Note 543, June 1998

[15] C. E. Baum, N. Geng, and L. Carin, "Integral equations and polarizability for magnetic singularity identification," Air Force Res. Lab., Kirtland AFB, NM, Interaction Note 524, Mar. 1997.

[16] S. Wakao and T. Onuki, "Electromagnetic field computations by the hybrid FE-BE method using edge elements," IEEE Trans. Magn., vol. 29, pp. 1487-1490, Feb. 1993.

[17] H. T. Yu and K. R. Shao, "H method for solving 3D eddy current problems," IEEE Trans. Magn., vol. 31, May 1995.

[18] A. Bossavit and I. D. Mayergoyz, "Edge elements for scattering problems," IEEE Trans. Magn., vol. 25, pp. 2816-2821, Apr. 1989.

[19] L. Collins, P. Gao, and L. Carin, "An improved Bayesian decision theoretic approach for land mine detection," IEEE Trans. Geosci. Remote Sensing, vol. 37, pp. 811-819, Mar. 1999.

Lawrence Carin (SM'96-F'01) was born March 25, 1963, in Washington, DC He received the B.S., M.S., and Ph.D. degrees in electrical engineering from the University of Maryland, College Park, in 1985, 1986, and 1989, respectively.

In 1989, he joined the Electrical Engineering Department, Polytechnic University, Brooklyn, NY, as an Assistant Professor, and became an Associate Professor there in 1994. In September 1995, he joined the Electrical Engineering Department at Duke University, Durham, NC, where he is now a Professor. He is the Principal Investigator on a Multidisciplinary University Research Initiative (MURI) on demining. His current research interests include short-pulse scattering, subsurface sensing, and wave-based signal processing.

Dr. Carin is a member of Tau Beta Pi and Eta Kappa Nu and is currently an Associate Editor of the IEEE TRANSACTIONS ON ANTENNAS AND PROPAGATION.

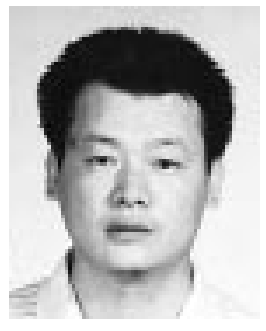

Haitao Yu was born in China in 1965. He receive the $\mathrm{Ph} . \mathrm{D}$. degree in electrical engineering from Huazhong University of Science and Technology (HUST), China, in 1995. From 1995 to 1997, he worked as postdoctoral fellow in the Department of Electrical Engineering, HUST. In 1997, he joined the same department as an Associate professor. From 1998, he was with Duke University, Durham, NC. His current research interests include wideband electromagnetic computation and numerical techniques for high and low frequencies.
Yacine Dalichaouch received the B.S. in physics from the University of Constantine, Algeria, and the Ph.D. in physics from the University of California, San Diego, in 1988, where he fabricated and investigated the magnetic and superconducting properties of highly correlated electron materials

Since 1996, his work has concentrated on the development of sensitive magnetic sensors and their applications in nondestructive evaluation of metal structures and detection/characterization of magnetic targets. He is currently a Senior Scientist and Magnetics Group Leader with Quantum Magnetics, Inc., San Diego, CA. He has close to 100 publications.

Dr. Dalichaouch is a member of the SPIE and APS.

Alexander R. Perry (M'96-SM'00) received the B.A. and Ph.D. degrees in engineering from Cambridge University, Cambridge, U.K., in 1988 and 1992, respectively, where he used cryogenic magnetometers for an investigation of the flux creep properties of high temperature superconducting materials.

Since 1992, his career has focused on applications of magnetic-field sensing using innovative configurations of both superconducting and room-temperature sensors. Currently, he serves as Group Leader for Advanced Systems with Quantum Magnetics, Inc., San Diego, CA, on research and development programs for data acquisition systems for a variety of magnetic sensing technologies.

Peter V. Czipott received the B.A. degree in physics and the Ph.D. degree from the University of California, San Diego, in 1975 and 1983, respectively, where he used superconducting gravimeters for geophysical studies and for an investigation of possible non-Newtonian gravitation at short distance scales.

Since 1993, his career has focused on applications of magnetic-field sensing, using innovative configurations of both superconducting and room-temperature sensors. Currently, he serves as Research Applications Manager with Quantum Magnetics, Inc., San Diego, CA, where he oversees the company's research and development programs outside the area of chemically specific explosives detection.

Dr. Czipott is a member of the APS, AAAS, AGU and SPIE.

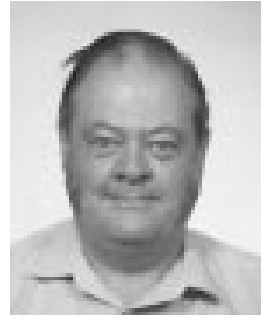

Carl E. Baum (S'62-M'63-SM'78-F'84) was born in Binghamton, NY, on February 6, 1940. He received the B.S. (with honors), M.S., and Ph.D. degrees in electrical engineering from the California Institute of Technology, Pasadena, in 1962, 1963, and 1969, respectively.

He was stationed at the Air Force Research Laboratory, Directed Energy Directorate (formerly Phillips Laboratory, formerly Air Force Weapons Laboratory), Kirtland AFB, Albuquerque, NM, from 1963 to 1967 and from 1968 to 1971 . Since 1971 , has served as a civil servant with a position as Senior Scientist, Air Force Research Laboratory. He is editor of several interagency note series on EMP (electromagnetic pulse) and related subjects and has published four books: Transient Lens Synthesis: Differential Geometry in Electromagnetic Theory, Electromagnetic Symmetry, Ultra-Wideband, Short-Pulse Electromagnetics 3 , and Detection and Identification of Visually Obscured Targets. He has led an EMP short course and HPE workshops at numerous locations around the globe.

Dr. Baum has been awarded the Air Force Research and Development Award (1970), the AFSC Harold Brown Award (1990), and Air Force Research Laboratory Fellow (1996). He has received the Richard R. Stoddart award (1984) of the IEEE EMC Society. He is a recipient of the 1987 Harry Diamond Memorial Award, one of the IEEE Field Awards, with citation "for outstanding contributions to the knowledge of transient phenomena in electromagnetics." $\mathrm{He}$ is a member of Commissions A, B, and E of the U.S. National Committee of the International Union of Radio Science (URSI) and is Founder and President of SUMMA Foundation, which sponsors various electromagnetics-related activities including scientific conferences, publications, short courses, fellowships, and awards. 